CERN-TH.7412/94

HD-HEP-94-30

OUTP-94

STUAM-FTUAM.40/94

\title{
NEUTRINO MASSES FROM GAUGE SYMMETRIES
}

\section{H. Dreiner ${ }^{1}$, G.K. Leontaris ${ }^{2}$, S. Lola ${ }^{3}$, Graham G. Ross, ${ }^{4, *}$ and C. Scheich ${ }^{5}$}

\author{
${ }^{1}$ ETH Hönggerberg, 8093 Zürich, Switzerland \\ ${ }^{2}$ CERN, Theory Division, 1211 Geneva 23, Switzerland, and \\ Physics Department, Ioannina University, Ioannina, Greece \\ ${ }^{3}$ Institut für Theoretische Physik, Univerisität Heidelberg, \\ Philosophenweg 16, 69120 Heidelberg, Germany \\ ${ }^{4}$ Department of Physics, Theoretical Physics, University of Oxford, \\ 1 Keble Road, Oxford OX1 3NP \\ ${ }^{5}$ Departemento de Física Teórica, Universidad Autónoma de Madrid, \\ 28049, Madrid, Spain
}

\begin{abstract}
A very simple extension of the Standard Model to include an Abelian family symmetry is able to describe the hierarchy of quark and lepton masses and their mixing angles together with the unification of gauge couplings. We consider the implications of this model for neutrino masses and mixing angles and show that they are determined up to a discrete ambiguity corresponding to the representation content of the Higgs sector responsible for the Majorana mass matrix.
\end{abstract}

* SERC Senior Fellow.

CERN-TH.7412/94

HD-HEP-94-30

OUTP-94

STUAM-FTUAM.40/94

August 1994 


\section{Introduction}

If we are to understand the pattern of fermion masses and mixing angles it is necessary to go beyond the Standard Model. One obvious possibility is that there is further structure, Grand Unification, strings etc., at a high scale which determines the Yukawa couplings responsible for the masses. However attempts to implement such ideas have to explain why the electroweak breaking scale is much less than the unification scale. Low energy supersymmetry [1] provides a way of protecting such an hierarchy of mass scales and if supersymmetry is broken by a gaugino condensate it can even explain the origin of the hierarchy.

Within the context of supersymmetry, unification [2, 3] has had considerable success in determining the parameters of the Standard Model «4. The measured values of the gauge couplings are consistent with simple unification values with a unification scale of $\mathrm{O}\left(10^{16} \mathrm{GeV}\right)$ provided the Standard Model spectrum is extended to that of the minimal supersymmetric model (the MSSM). In addition the pattern (and magnitude) of spontaneous breakdown of the Standard Model follows naturally from the structure of radiative corrections in the MSSM provided there is some unification of the supersymmetry breaking masses at the unification scale[2, 5]. This simplicity in the parameters of the (supersymmetric) Standard Model at high scales appears to extend to the Yukawa couplings involved in determining the fermion masses. The measured values of the bottom quark and the $\tau$ lepton masses are consistent with their equality at the unification scale [6, 4]. Further the mixing angles and masses have values consistent with the appearance of "texture" zeros in the mass matrix [0, 8, 9, 10], such texture zeros indicating the appearance of additional symmetries beyond the Standard Model . This, and the hierarchical structure observed in the quark and lepton mass matrices, strongly suggests the existence of an underlying family symmetry with breaking characterised by a small parameter, $\lambda[11$, 12]. In the limit the symmetry is exact only the third generation is massive and all mixing angles are zero. Symmetry breaking terms gradually fill in the mass matrices in powers of $\lambda$ generating an hierarchy of mass scales and mixing angles. Thus a broken symmetry can explain not only the "texture" zeros but also the relative magnitude of the non-zero elements.

It proves remarkably easy to construct such a broken symmetry giving rise to a realistic mass matrix. A simple Abelian gauge family symmetry spontaneously broken close to the Planck scale generates all the observed structure in the mass matrices. The structure is consistent with a much larger symmetry suggesting further unification, namely left-right-symmetry giving symmetric mass matrices, $S U(2)_{R}$ symmetry relating up and down quark couplings and a down- quark lepton symmetry 円 In ad-

\footnotetext{
${ }^{1}$ Such an extended symmetry would be consistent with $(S U(3))^{3}$ or $E_{6}$ gauge unification
} 
dition, consistency of this fermion mass structure with freedom of anomalies yields the successful prediction $\sin ^{2}\left(\theta_{W}\right)=3 / 8$ at the unification scale even though the gauge group is not Grand Unified 13.

In this letter we consider the implications of this scheme for neutrino masses and mixing angles in the case that the minimal multiplet content of the MSSM is extended to include right-handed neutrino components (plus the Standard Model singlet Higgses needed to generate their masses and to break the extended gauge family symmetry). Although this extends the multiplet content of the Standard Model it represents perhaps the most likely departure from the original Standard Model if neutrinos prove to have masses. The introduction of this sector requires the introduction of a Majorana mass matrix which has no counterpart in the Standard Model. Most attempts to deal with this matrix use very simple but ad hoc assumptions about its structure 14. We will show that, within the context of an extended family gauge symmetry with symmetric mass matrices, the structure of the Majorana mass matrix is determined up to a discrete ambiguity and that the resultant form is different from the usual assumptions made about its form. We will be particularly concerned to determine whether the pattern of light neutrino masses and mixings resulting from this structure can explain any of the experimental indications of neutrino mass.

\section{Quark and Charged Lepton Fermion Masses}

We start by reviewing the construction of the model[12] of quark and charged lepton masses. The structure of the mass matrices is determined by a family symmetry, $U(1)_{F D}$, with in general non-integer charge assignment of the Standard Model states

given in Table 1. The need to preserve $S U(2)_{L}$ invariance requires (left-handed) up and down quarks (leptons) to have the same charge. This plus the requirement of symmetric matrices then requires that all quarks (leptons) of the same i-th generation transform with the same charge $\alpha_{i}\left(a_{i}\right)$. The full anomaly free Abelian group involves an additional family independent component, $U(1)_{F I}$, and with this freedom we may make $U(1)_{F D}$ traceless without any loss of generality. Thus $\alpha_{3}=-\left(\alpha_{1}+\alpha_{2}\right)$ and $a_{3}=-\left(a_{1}+a_{2}\right)$.

The $U(1)_{F D}$ charge of the quark-antiquark pair has the form

$$
\left(\begin{array}{ccc}
-2\left(\alpha_{1}+\alpha_{2}\right) & -\alpha_{1} & -\alpha_{2} \\
-\alpha_{1} & 2 \alpha_{2} & \alpha_{1}+\alpha_{2} \\
-\alpha_{2} & \alpha_{1}+\alpha_{2} & 2 \alpha_{1}
\end{array}\right)
$$

but not with $S U(5)$. 


\begin{tabular}{|l|cccccccc|}
\hline & $Q_{i}$ & $u_{i}^{c}$ & $d_{i}^{c}$ & $L_{i}$ & $e_{i}^{c}$ & $\nu_{i}^{c}$ & $H_{2}$ & $H_{1}$ \\
\hline$U(1)_{F D}$ & $\alpha_{i}$ & $\alpha_{i}$ & $\alpha_{i}$ & $a_{i}$ & $a_{i}$ & $a_{i}$ & $-2 \alpha_{1}$ & $-2 \alpha_{1}$ \\
\hline
\end{tabular}

Table 1: $U(1)_{F D}$ symmetries.

This matrix neatly summarises the allowed Yukawa couplings for a Higgs boson coupling in a definite position. They should have charge minus that shown for the relevant position.

For the leptons we have a similar structure of lepton-antilepton charges

$$
\left(\begin{array}{ccc}
-2\left(a_{1}+a_{2}\right) & -a_{1} & -a_{2} \\
-a_{1} & 2 a_{2} & a_{1}+a_{2} \\
-a_{2} & a_{1}+a_{2} & 2 a_{1}
\end{array}\right)
$$

If the light Higgs, $H_{2}, H_{1}$, responsible for the up and down quark masses respectively have $U(1)$ charge so that only the $(3,3)$ renormalisable Yukawa coupling to $H_{2}, H_{1}$ is allowed, only the $(3,3)$ element of the associated mass matrix will be non-zero as desired. The remaining entries are generated when the $U(1)$ symmetry is broken. We assume this breaking is spontaneous via Standard Model singlet fields, $\theta$, $\bar{\theta}$, with $U(1)_{F D}$ charge $-1,+1$ respectively, which acquire equal vacuum expectation values (vevs) along a "D-flat" direction'2. After this breaking all entries in the mass matrix become nonzero. For example, the $(3,2)$ entry in the up quark mass matrix appears at $O\left(\epsilon^{\left|\alpha_{2}-\alpha_{1}\right|}\right)$ because $\mathrm{U}(1)$ charge conservation allows only a coupling $c^{c} t H_{2}\left(\theta / M_{2}\right)^{\alpha_{2}-\alpha_{1}}, \alpha_{2}>\alpha_{1}$ or $c^{c} t H_{2}\left(\bar{\theta} / M_{2}\right)^{\alpha_{1}-\alpha_{2}}, \alpha_{1}>\alpha_{2}$ and we have defined $\epsilon=\left(<\theta>/ M_{2}\right)$ where $M_{2}$ is the unification mass scale which governs the higher dimension operators. As discussed in reference [12] one may expect a different scale, $M_{1}$, for the down quark mass matrices (it corresponds to mixing in the $H_{2}, H_{1}$ sector with $M_{2}, M_{1}$ the masses of heavy $H_{2}$, $H_{1}$ fields). Thus we arrive at mass matrices of the form (suppressing unknown Yukawa couplings and their phases which are all expected to be of order 1)

$$
\begin{gathered}
\frac{M_{u}}{m_{t}} \approx\left(\begin{array}{ccc}
\epsilon^{|2+6 a|} & \epsilon^{|3 a|} & \epsilon^{|1+3 a|} \\
\epsilon^{|3 a|} & \epsilon^{2} & \epsilon^{1} \\
\epsilon^{|1+3 a|} & \epsilon^{1} & 1
\end{array}\right) \\
\frac{M_{d}}{m_{b}} \approx\left(\begin{array}{ccc}
\bar{\epsilon}^{|2+6 a|} & \bar{\epsilon}^{|3 a|} & \bar{\epsilon}^{|1+3 a|} \\
\bar{\epsilon}^{|3 a|} & \bar{\epsilon}^{2} & \bar{\epsilon}^{1} \\
\bar{\epsilon}^{|1+3 a|} & \bar{\epsilon}^{1} & 1
\end{array}\right)
\end{gathered}
$$

\footnotetext{
${ }^{2}$ The spontaneous breaking of gauge symmetries at high scales in supersymmetric theories must proceed along such flat directions to avoid large vacuum energy contributions from Dterms, giving $<\theta>=<\bar{\theta}>$.
} 
where $\bar{\epsilon}=\left(\frac{<\theta>}{M_{1}}\right)^{\left|\alpha_{2}-\alpha_{1}\right|}, \epsilon=\left(\frac{<\theta>}{M_{2}}\right)^{\left|\alpha_{2}-\alpha_{1}\right|}$ and $a=\alpha_{1} /\left(\alpha_{2}-\alpha_{1}\right)$. With $a=1$ the mass matrices are in excellent agreement with the measured values. We also point out that to a good approximation we have the relation [12]

$$
\epsilon=\bar{\epsilon}^{2}
$$

which also implies that $M_{2}>M_{1}$. This relation will be very helpful below when determining the structure of the neutrino spectrum.

The charged lepton mass matrix may similarly be determined. Requiring the good relation $m_{b}=m_{\tau}$ at unification sets $\alpha_{1}=a_{1}$ and we get

$$
\frac{M_{l}}{m_{\tau}} \approx\left(\begin{array}{ccc}
\bar{\epsilon}^{|2+6 a-2 b|} & \bar{\epsilon}^{|3 a|} & \bar{\epsilon}^{|1+3 a-b|} \\
\bar{\epsilon}^{|3 a|} & \bar{\epsilon}^{|2(1-b)|} & \bar{\epsilon}^{|1-b|} \\
\bar{\epsilon}^{|1+3 a-b|} & \bar{\epsilon}^{|1-b|} & 1
\end{array}\right)
$$

where $b=\left(\alpha_{2}-a_{2}\right) /\left(\alpha_{2}-\alpha_{1}\right)$. We will consider two choices for $b$ which give reasonable lepton masses.

For $b=0$ the lepton charges are the same as the down quark sector, and so the structure of the down quark and lepton mass matrices are identical. In order to explain the detailed difference between down quark and lepton masses it is necessary in this case to assume that the constants of proportionality determined by Yukawa couplings which we have so far taken to be equal (and of $\mathrm{O}(1)$ ) differ sightly for the lepton case. A factor 3 in the $(2,2)$ entry is sufficient to give excellent charged lepton masses.

An alternative which does not rely on different Yukawa couplings is to choose $b$ half integral. In this case the form for the lepton mass matrix, eq(6) must be modified and has the form

$$
M_{l}=\left(\begin{array}{ccc}
\bar{\epsilon}^{|2+6 a-2 b|} & \bar{\epsilon}^{|3 a|} & 0 \\
\bar{\epsilon}^{|3 a|} & \bar{\epsilon}^{|2(1-b)|} & 0 \\
0 & 0 & 1
\end{array}\right)
$$

The zeros in this mass matrix result because there is now a residual $Z_{2}$ discrete gauge symmetry after $U(1)$ breaking by which the electron and muon fields get transformed by a factor $(-1)$. For $a=1, b=1 / 2$ we get excellent agreement for the charged lepton masses; for this choice one gets the following relations at laboratory energies

$$
\begin{aligned}
& m_{\mu} \approx \frac{m_{s}}{3 \bar{\epsilon}} \\
& m_{e} \approx \frac{m_{d} \bar{\epsilon}}{3}
\end{aligned}
$$

where the factor 3 is due to the renormalisation group corrections found when continuing from the Grand Unification scale to the laboratory scale. This is in good agreement with the experimentally measured values for the value $\bar{\epsilon}=0.23$ needed to fit the down quark masses and mixing angles. Using Eq.(5) this in turn implies that $\epsilon=0.053$. 


\section{$3 \quad$ Neutrino Mass Matrices}

We turn now to a discussion of the implications of this broken symmetry for neutrino masses in the case we add three generations of right-handed neutrinos. Note first that $S U(2)_{L}$ fixes the $U(1)_{F D}$ charge of the left-handed neutrino states to be the same as the charged leptons. The left- right- symmetry then fixes the charges of the right-handed neutrinos as given in Table 1. Thus we have no freedom in assigning right-handed neutrino charges and as a result the neutrino Dirac mass is also fixed to be

$$
\frac{M_{\nu_{R}}^{D}}{m_{\nu_{\tau}}} \approx\left(\begin{array}{ccc}
\epsilon^{|2+6 a-2 b|} & \epsilon^{|3 a|} & \epsilon^{|1+3 a-b|} \\
\epsilon^{|3 a|} & \epsilon^{|2(1-b)|} & \epsilon^{|1-b|} \\
\epsilon^{|1+3 a-b|} & \epsilon^{|1-b|} & 1
\end{array}\right)
$$

for $b$ integer or

$$
\frac{M_{\nu}^{D}}{m_{\nu_{\tau}}} \approx\left(\begin{array}{ccc}
\epsilon^{|2+6 a-2 b|} & \epsilon^{|3 a|} & 0 \\
\epsilon^{|3 a|} & \epsilon^{|2(1-b)|} & 0 \\
0 & 0 & 1
\end{array}\right)
$$

for $b$ half integer. Thus the Dirac neutrino mass matrices are related in scale to the up-quark mass matrices, similar to models based on Grand Unified Theories.

Of course the mass matrix structure of neutrinos is more complicated due to the possibility of Majorana masses for the right-handed components]. The right handed fields on the other hand can get Majorana masses from a term of the form $\nu_{R} \nu_{R} \Sigma$ where $\Sigma$ is a $S U(3) \otimes S U(2) \otimes U(1)$ invariant Higgs scalar field with $I_{W}=0$ and $\nu_{R}$ is a righthanded neutrino. In many models $\Sigma[15]$ is a combination of scalar fields $\Sigma=\tilde{\bar{\nu}}_{R} \tilde{\bar{\nu}}_{R}$ where $\tilde{\bar{\nu}}_{R}$ is the scalar component of a right-handed antineutrino supermultiplet: $\bar{\nu}_{R}$. For definiteness we mostly focus on this model but our main results do not depend on this choice.

The structure of the resulting Majorana mass matrix depends on the $U(1)_{F D}$ invariant combinations contributing. Clearly the right-handed neutrino bilinears have the $U(1)_{F D}$ charge structure of eq(2). Thus the Majorana mass matrix, $M_{\nu}^{M}$, depends entirely on the $\Sigma U(1)_{F D}$ charge. The possible choices for it will give a discrete spectrum of possible forms for the Majorana mass.

For example if, in the absence of $U(1)_{F D}$ symmetry breaking the $\Sigma$ charge is the same as the $H_{1,2}$ doublet Higgs charges, only the $(3,3)$ element of $M_{\nu}$ will be non-zero. Allowing for $U(1)_{F D}$ breaking by $<\theta>$ the remaining elements in the Majorana mass matrix will be generated in an analogous way to the generation of the Dirac mass

\footnotetext{
${ }^{3}$ For the left-handed components these do not appear for the usual reason, namely the absence of $\Delta I_{W}=1$ mass terms coming from weak isospin $0\left(I_{W}=0\right)$ Higgs fields.
} 
matrices. If The important question is what is the appropriate expansion parameter? It will be given by $\eta=\frac{<\Theta>}{M_{3}}$, where $M_{3}$ is the scale determining the mixing in the $I_{W}=0$ sector. In the case of $H_{1}, H_{2}$ the scale was identified with the massive $H_{1}, H_{2}$ fields which mix with the light $H_{1}, H_{2}$ fields when $\langle\theta\rangle$ is non-zero. Likewise, in the $I_{W}=0$ Higgs sector $M_{3}$ should be identified with the massive $\Sigma$ modes. For the case that $\Sigma$ is the bilinear $\Sigma=\tilde{\bar{\nu}}_{R} \tilde{\bar{\nu}}_{R}$ it is the mixing of the $\bar{\nu}_{R}$ fields that generates the mixing and hence the mass of the intermediate $\bar{\nu}_{R}$ fields that is relevant.

What is the expectation for such masses? The pattern of masses generated here relies on the mixing of light and heavy Higgs fields. Consider a string compactification which in addition to $H_{1}$ and $H_{2}$, leaves additional Higgs multiplets $H_{1,2}^{a, b \ldots}, \bar{H}_{1,2}^{a, b \ldots}$ light. The pairs of Higgs fields in conjugate representations can have gauge invariant masses and may be expected to become massive if there is any stage of spontaneous symmetry breaking below the compactification scale with a common mass, $M$, where $M=<$ $\Phi>$ and $\Phi$ is a gauge invariant Higgs scalar field (or a gauge invariant combination of Higgs fields). There may be further sources of Higgs mass. As we have stressed our left- right- symmetry essentially requires an extension of the gauge symmetry to $S U(2)_{L} \otimes S U(2)_{R}$ at high scales. This will be broken by a right-handed sneutrino vev in which case the mass degeneracy of the $H_{1}$ and $H_{2}$ pair which transform as a $(1 / 2,1 / 2)$ representation under $S U(2)_{L} \otimes S U(2)_{R}$ can be split via the coupling $<\tilde{\nu}_{R}>H_{2} H_{x}$ where $H_{x}$ transforms as $(1 / 2,0)$. Such a contribution will generate $M_{2} \approx<\tilde{\nu}_{R}>$, $M_{1} \approx M$ agreeing well with Eq.(5) and the phenomenological findings of [12].

Turning to the expectation for $\nu_{R}$ masses we may expect the $\bar{\nu}_{R}$ fields to aquire mass of $O\left(M_{1}\right)$ via a $\Phi \nu \bar{\nu}$ coupling. These fields may also acquire a mass via the term $<\tilde{\nu}_{R}>^{2}\left(\bar{\nu}_{R}\right)^{2}$ giving a mass $<\tilde{\nu}_{R}>^{2} / M_{P l}$. Given that $<\tilde{\nu}_{R}>\approx M_{2}$ then $\epsilon=\bar{\epsilon}^{2}$ from Eq.(5) implies that $<\tilde{\nu}_{R}>^{2} / M_{P l}=M_{1} \cdot\left(M_{2} / M_{P l}\right) / \bar{\epsilon}$. To preserve the successful relations between up and down quarks we require that non - renormalisable contributions to the up quark masses should be less than that coming from the mixing with the heavy $H_{2}$ fields and so $M_{2} / M_{P l}<<1$. Thus these terms are not expected to spoil our estimate that $m_{\nu_{R}} \approx M_{1}$.

This implies that the appropriate expansion parameter for the Majorana mass matrix is the same as that for the down quarks and charged leptons, namely $\eta \approx \bar{\epsilon}$. In a specific model this relation can be determined exactly. In general we only expect this to hold up to factors of order one.

We may now compute the patterns of Majorana mass for the different possible choices of $\Sigma$ charge. These are given in Tables 2 and 3 together with the mass eigen-

\footnotetext{
${ }^{4}\langle\Sigma\rangle$ is significantly below the Planck scale and thus $\langle\theta\rangle$ dominates the $U(1)_{F D}$ breaking.
} 
values. We have made use of Eq.(5). Note the result is quite different from the usual ansatz which assumes it is family independent. In fact none of the possibilities of Table 2 give a family independent structure for the Majorana mass. This is perhaps the most important lesson to be derived from this model; if symmetries are responsible for the hierarchical pattern of fermion masses they are very likely to generate an hierarchical pattern for the Majorana mass matrix. Moreover, as may be seen from Table 2, in most of the cases the lighter $M_{\nu_{R}}$ eigenstate is suppressed by several powers of $\bar{\epsilon}$ compared to the heavier one. The implications for the phenomenology of neutrino masses is quite different as we shall now discuss.

Of relevance to low energy phenomena is the pattern of light neutrino masses and mixing angles. To compute these we must determine the light neutrino mass matrix, $M_{\nu}^{e f f}$, coming from the "see-saw" mechanism. It is given by

$$
M_{\nu}^{e f f}=M_{\nu}^{D} \cdot\left(M_{\nu_{R}}^{M}\right)^{-1} \cdot M_{\nu}^{D}
$$

Using the two forms for the neutrino Dirac matrices given in eqs(9) and (10) together with the appropriate possibilities for the Majorana mass matrix, Tables 2 and 3 respectively, we may compute the masses and mixing angles for the light neutrinos. These are given in Tables 4 and 5 respectively. The use of Eq.(5) greatly simplifies the results.

As mentioned above, in no case does the light Majorana mass matrix have degenerate eigenvalues, the most common assumption made for its structure. The reason is that the gauge symmetry charges of the right-handed neutrinos force the mass matrix entries to be of different orders in powers of the expansion parameter $\epsilon$. The only way a degenerate set of eigenvalues can occur in this case is if two components are coupled through an off diagonal mass term as in cases 2, 4, 5 and 9. As may be seen this only leads to two of the three eigenvalues being approximately equal. The implications of this for the light neutrinos is clear from Tables 4 and 5 . In no case are the mass eigenvalues simply given in terms of the square of the up quark masses divided by a common Majorana mass (the usual ansatz, giving the ratio $1: \bar{\epsilon}^{2}: \bar{\epsilon}^{4}$ ). Rather the effect of structure in the Majorana masses is to allow for a greater spread in masses. As may be seen from these Tables there is no example of closely degenerate pairs of neutrino masses; clearly this is of considerable significance for neutrino oscillation phenomenology. In particular all three hints for neutrino masses (solar neutrino problem, atmospheric neutrino problem, and the structure formation problem, requiring a one third component of hot dark matter [17]) taken at face value can only be resolved in agreement with collider bounds (without the use of additional sterile neutrinos) for three nearly degenerate neutrinos of approximate mass $2-3 \mathrm{eV}$ [18. This can not be accomodated in our scheme without fine tuning. However, as we discuss below, we can find simultaneous solutions to the solar neutrino problem and the neutrino mass 


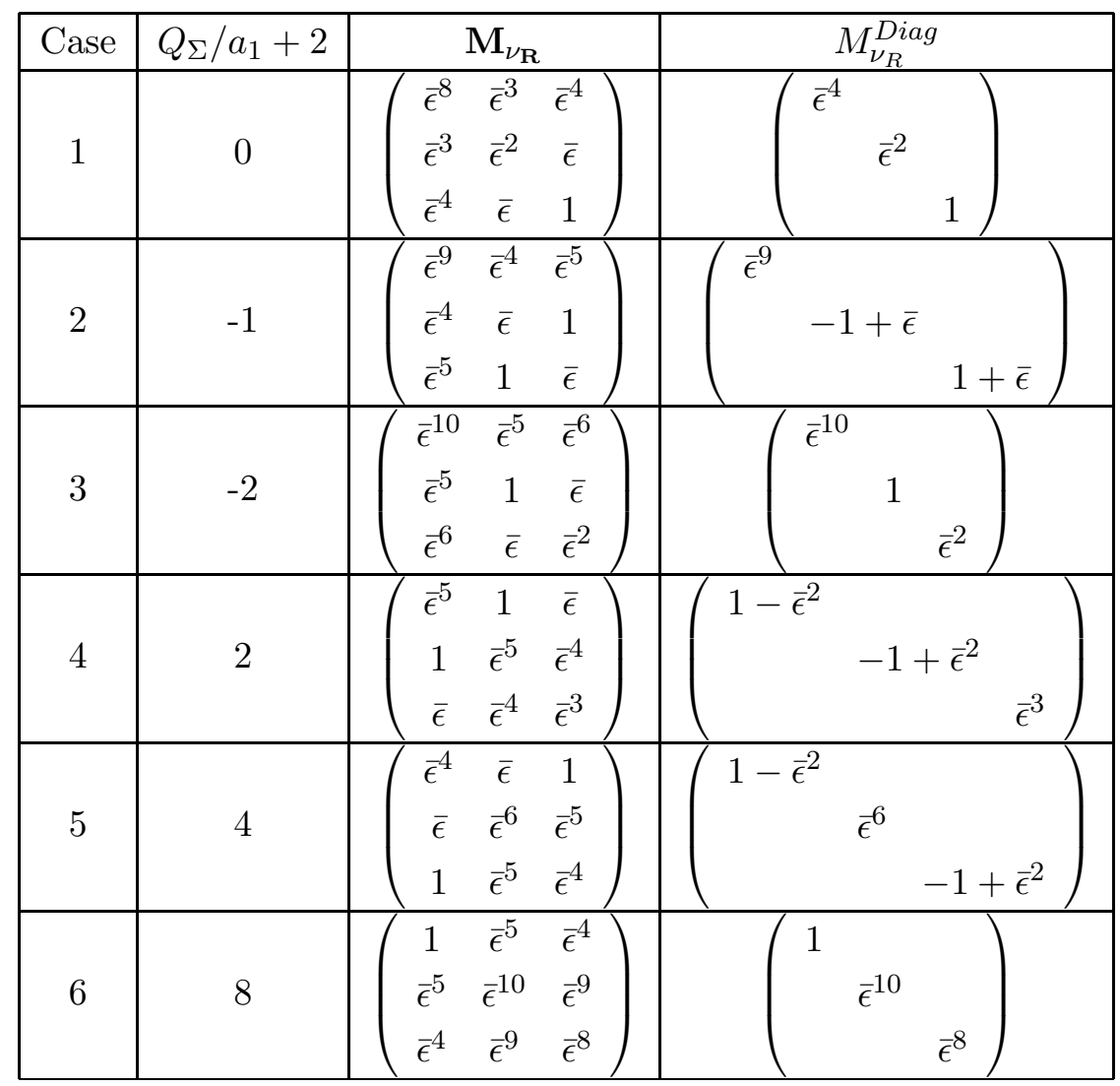

Table 2: Majorana mass matrix, $M_{\nu_{R}}$, for the right-handed neutrinos for a range of choices of $Q_{\Sigma}$, the $U(1)$ charge of the $I_{W}^{R}=2$ effective Higgs field. These cases correspond to $b=0$ and $a=1$ in eq(6). Note that we have suppressed all coefficients of $\mathrm{O}(1)$. For example in case 1 the $(2 \otimes 2)$ submatrix comprising the second and third rows and columns does not have vanishing determinant for random choices of these coefficients and the mass eigenvalues quoted refer to such generic cases. 


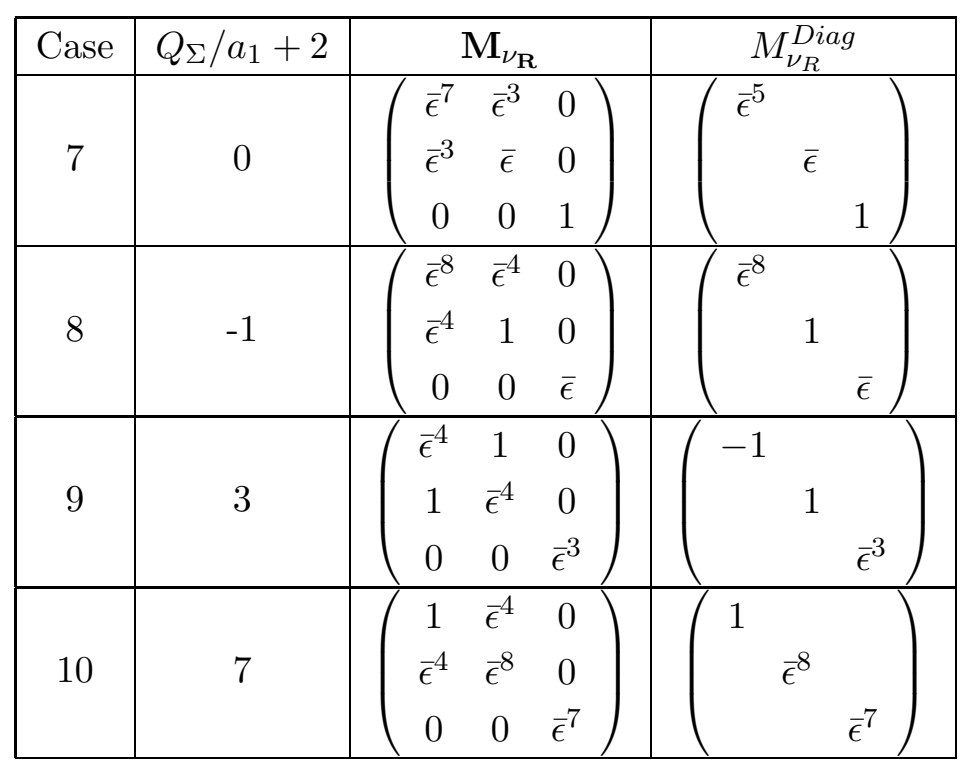

Table 3: Majorana mass matrix, $M_{\nu_{R}}^{M}$, for the right-handed neutrinos for a range of choices of $Q_{\Sigma}$, the $U(1)$ charge of the $I_{W}^{R}=2$ effective Higgs field. These cases correspond to $b=1 / 2, a=1$ in eq(6).

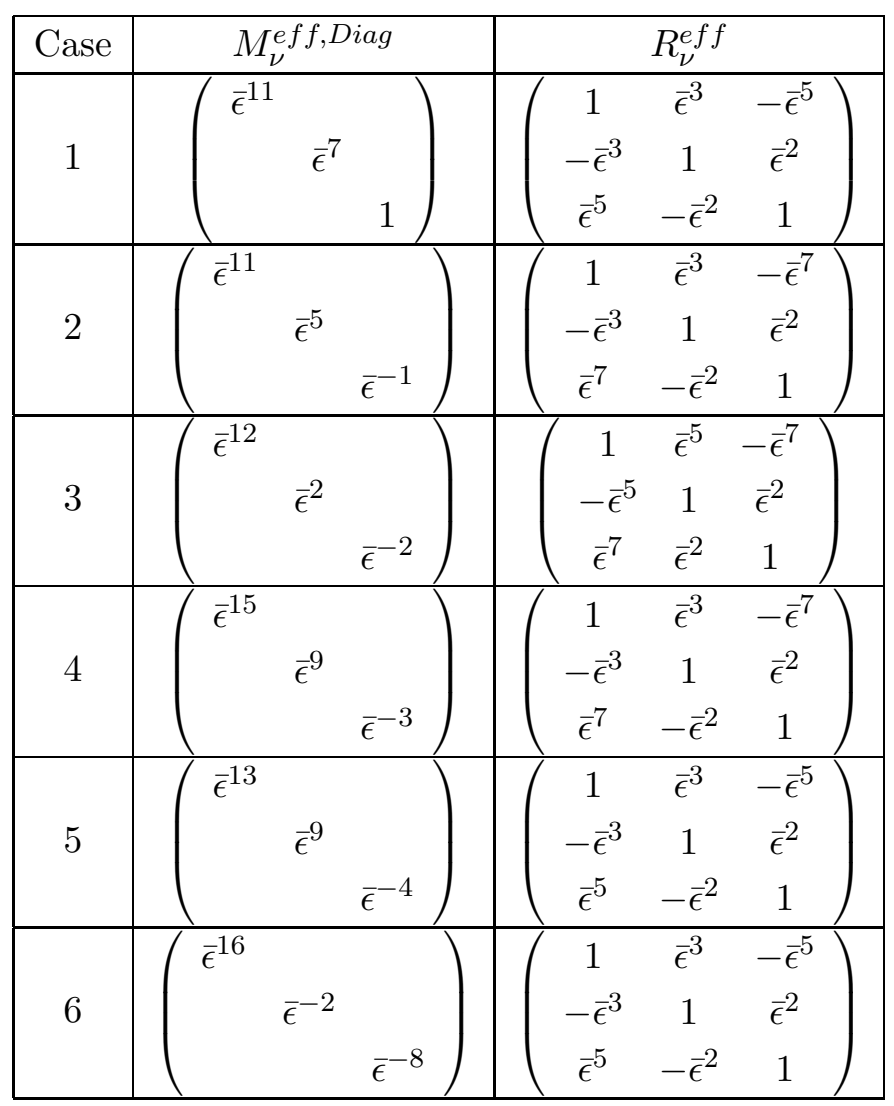

Table 4: Masses and mixing angles for the light neutrino components. 


\begin{tabular}{|c|c|c|c|}
\hline Case & $M_{\nu}^{\text {eff,Diag }}$ & $R_{\nu}^{\text {eff }}$ \\
\hline 7 & $\left(\begin{array}{ccc}\bar{\epsilon}^{13} & & \\
& \bar{\epsilon}^{5} & \\
& & 1\end{array}\right)$ & $\left(\begin{array}{ccc}1 & \bar{\epsilon}^{4} & 0 \\
-\bar{\epsilon}^{4} & 1 & 0 \\
0 & 0 & 1\end{array}\right)$ \\
\hline 8 & $\left(\begin{array}{ccc}\bar{\epsilon}^{12} & & \\
& \bar{\epsilon}^{4} & \\
& & \bar{\epsilon}^{-1}\end{array}\right)$ & $\left(\begin{array}{ccc}1 & \bar{\epsilon}^{4} & 0 \\
-\bar{\epsilon}^{4} & 1 & 0 \\
0 & 0 & 1\end{array}\right)$ \\
\hline 9 & $\left(\begin{array}{ccc}\bar{\epsilon}^{16} & & \\
& \bar{\epsilon}^{8} & \\
& & \bar{\epsilon}^{-3}\end{array}\right)$ & $\left(\begin{array}{ccc}1 & \bar{\epsilon}^{4} & 0 \\
-\bar{\epsilon}^{4} & 1 & 0 \\
0 & 0 & 1\end{array}\right)$ \\
\hline 10 & $\left(\begin{array}{ccc}\bar{\epsilon}^{20} & & \\
& \bar{\epsilon}^{-4} & \\
1 & \bar{\epsilon}^{4} & 0 \\
-\bar{\epsilon}^{4} & 1 & 0 \\
0 & 0 & 1\end{array}\right)$ \\
\hline
\end{tabular}

Table 5: Masses and mixing angles for the light neutrino components.

needed for structure formation.

\section{$4 \quad$ Neutrino Mixing Angles}

The second point of interest are the predictions for mixing angles in the light neutrino sector. It may be seen that the consequence of the gauge symmetry is to generate mixing angles which are small, given by some power of the expansion parameter $\bar{\epsilon}$. Of course the mixing angles of relevance to experiment are the combination of charged lepton mixing angles and neutrino mixing angles given by $R_{\text {total }}^{\text {eff }}=\left(R^{\text {eff }}\right)_{\nu}^{-1} R_{L}$, where

$$
R_{L}=\left(\begin{array}{ccc}
1 & \delta_{e \mu} & O\left(\bar{\epsilon}^{4}\right) \\
-\delta_{e \mu} & 1 & O(\bar{\epsilon}) \\
-O\left(\bar{\epsilon}^{4}\right) & -O(\bar{\epsilon}) & 1
\end{array}\right)
$$

for the charged lepton mass matrix of eq(6) with $b=0$ and $a=1$. Here, due to the $(1,1)$ approximate "texture" zero $\delta_{e \mu}=\sqrt{m_{e} / m_{\mu}}$. For the charged lepton mass matrix of eq(7) with $b=1 / 2, a=1$ we have

$$
R_{L}=\left(\begin{array}{ccc}
1 & \delta_{e \mu} & 0 \\
-\delta_{e \mu} & 1 & 0 \\
0 & 0 & 1
\end{array}\right)
$$

From Table 1 we may easily determine that the only effect of the neutrino mixing angles for cases $(1-6)$ is in the $(1,3)$ element of $R_{\text {total }}^{\text {eff }}$ where the term of order $\epsilon^{4}$ in $R_{L}$ 
becomes of order $\epsilon^{3}$ in $R_{\text {total }}^{\text {eff }}$. The remaining elements are the same as in $R_{L}$, eq(12) or eq(13), because of the smallness of the neutrino mixing angles.

The situation for the cases $(7-10)$ is simpler due to the residual $Z_{2}$ symmetry. For them in all cases the neutrino mixing angles are so small that $R_{\text {total }}^{e f f} \approx R_{L}$. In this case there is no mixing $\nu_{\mu}-\nu_{\tau}$. These cases could be excluded by a positive finding of the CHORUS and NOMAD experiments [19].

\section{Phenomenology}

Let us now discuss the implications for neutrino phenomenology following from these mass structures. Although we have determined the relative magnitude of the $M_{\nu}^{M}$ and $M_{\nu}^{e f f}$, we are of course interested in the expectation for their absolute magnitudes. This depends on the origin of the $I_{W}^{R}=2$ effective Higgs field, $\Sigma$. If $\Sigma=\tilde{\bar{\nu}}_{R} \tilde{\bar{\nu}}_{R}$ the Majorana masses of Tables 2 and 3 are given in units $<\tilde{\bar{\nu}}_{R}><\tilde{\bar{\nu}}_{R}>/ M$ where $\mathrm{M}$ is the mass scale governing the appearance of higher dimension operators, typically the string scale or $M_{\text {Planck. }}$. Given the success of the unification of gauge couplings at a scale of $O\left(10^{16} \mathrm{GeV}\right)$ it is reasonable to choose $<\tilde{\nu}_{R}>=O\left(10^{16} \mathrm{GeV}\right)$ leading to the scale $10^{13}-10^{14} \mathrm{GeV}$ for the Majorana mass scale. This in turn implies the unit of mass for the light neutrinos masses given in Tables (4. (4) is $(4-0.4) \mathrm{eV}$ for a top quark of $O(200) G e V$. This means that it is quite reasonable for the heaviest neutrino to have a mass of the magnitude needed for structure formation (i.e. of $O(10 \mathrm{eV})$ ) [17].

Remarkably, as we will now discuss, for a subset of the solutions the light neutrinos have masses and mixing angles of the magnitude needed to explain solar neutrino oscillations. The experimental data on solar neutrino observations may be explained if the masses and mixing angles of the neutrinos fall in the following ranges 20:

a) The small mixing angle solution for the MSW effect requires

$$
\begin{aligned}
\delta m_{\nu_{e} \nu_{\mu}}^{2} & \approx(0.6-1.2) \times 10^{-5} \mathrm{eV}^{2} \\
\sin ^{2} 2 \theta_{\mu e} & \approx(0.6-1.4) \times 10^{-2}
\end{aligned}
$$

b) Vacuum oscillations can solve the solar neutrino if

$$
\begin{aligned}
\delta m_{\nu_{e} \nu_{\mu}}^{2} & \approx(0.5-1.1) \times 10^{-10} \mathrm{eV}^{2} \\
\sin ^{2} 2 \theta_{\mu e} & \geq 0.75
\end{aligned}
$$

Consider the small mixing angle solution. If the lightest two neutrinos are to have masses in the range needed for the MSW explanation of the solar neutrino deficit we 
need a suppression factor of $O\left(10^{-3}\right)$ relative to the heaviest neutrino corresponding to $O\left(\bar{\epsilon}^{4}\right)$ for $\bar{\epsilon} \approx 0.23$. We see this is true for case 3 and is marginally consistent too with cases 7,8 and 10 . We may go further and consider whether the expectation for the mixing angle between the two lightest generations is in the range needed by the MSW effect. The mixing angle of relevance to the solar neutrino problem is $\left(R_{\text {total }}^{\text {eff }}\right)_{\mu e}$. In most of the cases presented in the tables the light neutrino mixing matrices exhibit negligible $\nu_{\mu}-\nu_{e}$ mixing and $\left(R_{\text {total }}^{e f f}\right)_{\mu e}$ is mainly determined by the charged lepton mixing angle $\sqrt{\frac{m_{e}}{m_{\mu}}}$. Although of the correct order of magnitude, in detail this mixing is larger than that required to solve the solar neutrino problem. However in cases 1, 2, 4,5 and 6 the contribution to $\nu_{\mu}-\nu_{e}$ mixing from the neutrino sector is not entirely negligible and we have

$$
\left(R_{\text {total }}^{e f f}\right)_{\mu e} \approx \sqrt{\frac{m_{e}}{m_{\mu}}}-O\left(\bar{\epsilon}^{3}\right)
$$

For $\bar{\epsilon}=0.23$ and taking the constant of proportionality to be unity, one gets $\sin ^{2} 2 \theta_{e \mu} \geq$ $1.310^{-2}$ which is within the limits of the mixing required to explain the solar neutrino problem.

The vacuum solution to the solar neutrino problem as well as the neutrino oscillation solution to the atmospheric neutrino problem require large mixing angles of $O(1)$. As may be seen from the Tables this is not to be expected in the $U(1)$ family symmetry presented here. Large mixing angles can be obtained but only by fine tuning of the Yukawa couplings. As we have stressed although we cannot rule out such a possibility we consider it unlikely as no symmetry requires such fine tuning.

Finally we comment on how one may choose between the ten different cases discussed above. As we have stressed they correspond to possible choices for the $\Sigma$ charge. Is this constrained? In [12] the constraints of anomaly freedom were used to limit the possible fermion mass matrix structures coming from the $U(1)_{F D}$. We would like to extend this to the case of interest here involving additional right-handed neutrinos and the $\Sigma$ field. However as these are $S U(3) \otimes S U(2)_{L} \otimes U(1)$ singlets they do not affect the anomaly structure discussed in [12]. If, however, the gauge group is extended 1 to $S U(3) \otimes S U(2)_{L} \otimes S U(2)_{R} \otimes U(1)$ there will be constraints on the $\Sigma$ charge. From the left- right- symmetry we may conclude that the contributions to the $S U(2)_{L}^{2} U(1)_{F D}$ and $S U(2)_{R}^{2} U(1)_{F D}$ anomalies are the same from the matter fields. In 12 the Higgs fields had to be neutral under $U(1)_{F D}$ to maintain anomaly cancellation (via the Green Schwarz mechanism[21]). The same consideration applied to the $\Sigma$ field requires $Q_{\Sigma}+2 a_{1}$ to be zero corresponding to the cases 1 and 7 . We note that these cases were consistent with neutrino masses generating structure and solar

\footnotetext{
${ }^{5}$ This seems inevitable if we are to explain the symmetric mass matrix structure assumed here.
} 
neutrino oscillations via the MSW effect. 


\section{References}

[1] For reviews see H. P. Nilles, Phys. Rep. 110 (1984) 1; G. G. Ross, Grand Unified Theories, Benjamin Cummings (1985); H.E. Haber and G.L. Kane, Phys. Rep. 117 (1985) 75.

[2] S. Dimopoulos and H. Georgi, Nucl. Phys. B 193 (1981) 150; S. Ferrara, ed. in 'Supersymmetry', (North Holland, Amsterdam, 1987); G. Costa, J. Ellis, G.L. Fogli, D.V. Nanopoulos and F. Zwirner, Nucl.Phys. B297 (1988) 244; J. Ellis, S. Kelley and D.V. Nanopoulos, Phys. Lett. B249 (1990)441; Phys. Lett. B260 (1991) 131; P. Langacker, Pennsylvania preprint UPR-0435T, (1990); U. Amaldi, W. de Boer and H. Fürstenau, Phys. Lett. B260 (1991) 447;P. Langacker and M. Luo, Phys.Rev. D44 (1991) 817; G.G. Ross and R.G. Roberts, Nucl. Phys. B 377 (1992) 571; F. Anselmo, L. Cifarelli, A. Peterman and A. Zichichi, Nuovo Cim. 104A (1991) 1817, ibid 104A(1992)1336.

[3] for recent works see e.g. G. L. Kane, C. Kolda, L. Roszkowski and J. D. Wells, Phys. Rev. D49 (1994) 49; M. Carena, M. Olechowski, S. Pokorski and C.E.M. Wagner, MPI preprints, MPI-Ph/93-66 and MPI-Ph/93-103; P. Langacker and N. Polonsky, Phys. Rev. D49 (1994) 1454 and UPR-0594T (1994) preprint and references therein.

[4] H. Arason, D.J. Castaño, B. Keszthelyi, S. Mikaelian, E.J. Piard, P. Ramond and B.D. Wright, Phys. Rev. Lett. 67 (1991) 2933; B. Anathanathan, G. Lazarides and Q. Shafi, Phys.Rev. D44 (1991)1613; A. Anderson et al, Phys. Rev. D 49 (1994)3660.

[5] K. Inoue et al., Prog. Theor. Phys. 68 (1982) 927; L.E. Ibáñez, Nucl.Phys. B218 (1983) 514; L.E. Ibáñez and C. López, Phys. Lett. B126 (1983) 54; Nucl.Phys. B233 (1984) 511; L. Alvarez-Gaume, J. Polchinsky and M. Wise, Nucl.Phys. B221 (1983) 495; L.E Ibáñez, C. López and C. Muñoz, Nucl. Phys. B256 (1985) 218.

[6] M.S. Chanowitz, J. Ellis and M.K. Gaillard, Nucl. Phys. B 128 (1977) 506. A. Buras, J. Ellis, M.K. Gaillard and D.V. Nanopoulos, Nucl. Phys. B 135 (1978) 66.

[7] S. Weinberg, in "A Festschrift for I.I. Rabi" [Trans. N.Y. Acad. Sci., Ser. II (1977), v. 38], p. 185; F. Wilczek and A. Zee, Phys. Lett. B 70 (1977) 418. T. Maehara and T. Yanagida, Prog. Theor. Phys. 60 (1978) 822 J. Chakrabarti, Phys. Rev. D20 (1979) 2411 F. Wilczek and A. Zee, Phys. Rev. Lett. 42 (1979) 421.

[8] H. Fritsch,Phys. Lett. B 70 (1977) 436; Phys. Lett. B 73 (1978) 317; F.J. Gilman and Y. Nir, Ann. Rev. Nucl. Part. Sci. 40 (1990) 213; P. Kaus and S. Meshkov, Mod. Phys. Lett. A3 (1988) 1251. 
[9] C.D.Froggat and H.B. Nielsen, Origin of symmetries, World Scientific (1991) N. Cabibbo, Phys. Rev. Lett. 10 (1963) 531 M. Kobayashi and T. Maskawa, Prog. Theor. Phys. 49 (1973) 652 M.E. Machacek and M.T. Vaughn, Phys. Lett. B103 (1981) 427.

[10] J. Harvey, P. Ramond and D. Reiss, Phys. Lett. B 92 (1980) 309; S. Dimopoulos, L. J. Hall and S. Raby, Phys. Rev. Lett. 68 (1992) 1984; Phys. Rev. D 45 (1992) 4195; H. Arason, D. J. Castaño, P. Ramond and E. J. Piard, Phys. Rev. D 47 (1993) 232; G. F. Giudice, Mod. Phys. Lett. A7 (1992) 2429.

[11] P.Ramond, R.G. Roberts and G.G. Ross, Nucl. Phys. B406 (1993) 19.

[12] L.E. Ibáñez and G.G. Ross, Phys. Lett. B 332 (1994)100.

[13] L. E. Ibáñez, Phys. Lett. B 303 (1993) 55.

[14] S. Dimopoulos et al, Phys. Rev. D47 (1993)R3697; H. Dreiner et al, Mod. Phys. Lett. A8 (1993) 2099; K. Babu and Q. Shafi, Phys.Lett. B311 (1993) 172; E. Papageorgiu, Orsay preprint, LPTHE-ORSAY-93-45.

[15] F. del Anguilla, G. Blair, M. Daniel and G.G. Ross, Nucl. Phys. B272 (1986)413; B.R. Green, K.H. Kirklin, P.J. Miron and G.G. Ross, Nucl. Phys. B292 (1987)606.

[16] L.E. Ibáñez, and G.G.Ross, in preparation.

[17] E.L. Wright et al., Astrophys. J.396 (1992) L13; M. Davis et al., Nature 359(1992) 393; A.N. Taylorand M. Rowan-Robinson, ibid 359 (1992) 396.

[18] S. Petcov and A. Smirnov, Phys.Lett. B322 (1994) 109; D. O. Caldwell, R. N. Mohapatra, UCSB-HEP-94-03; hep-ph-9402231.

[19] CHORUS collaboration, N. Armenise et al., CERN-SPSC/90-42 (1990); NOMAD collaboration, P. Astier et al., CERN-SPSC/91-21 (1991).

[20] see for example, L. Wolfenstein, Phys.Rev. D17 (1978) 20; S. P. Mikheyev and A. Yu Smirnov, Yad. Fiz.42 (1985) 1441; J. N. Bahcall and W.C. Haxton, Phys.Rev. D40 (1989) 931; X. Shi, D. N. Schramm and J. N. Bahcall, Phys.Rev.Lett. 69 (1992) 717; P. I. Krastev and S. Petcov, Phys.Lett. B299 (1993) 94; N. Hata and P. Langacker, Phys.Rev. D50 (1994) 632 and references therein.

[21] M. Green and J. Schwarz, Phys. Lett. B 149 (1984) 117; M. Dine, N. Seiberg and E. Witten, Nucl.Phys. B289 (1987) 585; J.Atick, L. Dixon and A. Sen, Nucl.Phys. B292 (1987) 109; M. Dine, I. Ichinoise and N. Seiberg, Nucl.Phys. B293 (1987) 253. 